\title{
Neuropathic pain models and outcome measures: a dual translational challenge
}

\author{
Robert D. E. Sewell \\ Cardiff School of Pharmacy and Pharmaceutical Sciences, Cardiff University, Cardiff, UK \\ Correspondence to: Robert D. E. Sewell. Cardiff School of Pharmacy and Pharmaceutical Sciences, Cardiff University, Cardiff CF10 3NB, UK. \\ Email: sewell@cardiff.ac.uk. \\ Comment on: Barragán-Iglesias P, Oidor-Chan VH, Loeza-Alcocer E, et al. Evaluation of the neonatal streptozotocin model of diabetes in rats: \\ Evidence for a model of neuropathic pain. Pharmacol Rep 2018;70:294-303.
}

Submitted Sep 17, 2018. Accepted for publication Sep 22, 2018.

doi: $10.21037 / \mathrm{atm} .2018 .09 .58$

View this article at: http://dx.doi.org/10.21037/atm.2018.09.58

The phenomenon of pain may be neuropathic in nature and it invariably stems from disease or injury affecting the somatosensory system $(1,2)$. Neuropathic pain is usually a chronic condition and is a significant cause of morbidity. It has a pronounced impact on wellbeing as well as patient quality of life and of those patients presenting with pain, up to a quarter of them suffer neuropathic pain $(3,4)$. It involves symptoms of an abnormally unpleasant sensation (dysesthesia), allodynia typified by a painful sensation to a normally innocuous stimulus and hyperalgesia characterized by an exaggerated pain perception to a normally noxious stimulus (5).

Hyperalgesia ensues from aberrant nociceptor input processing, whereas allodynia can be evoked by activity of low threshold myelinated $A \beta$ nerve fibres on a perturbed central nervous system and also a diminished threshold of peripheral nociceptor terminals (1). In this context, damage to peripheral nerves can lead to dysfunctional adaptation at different locations in the pain pathway and substantial changes in the central nervous system take place as a consequence. Hence, it has been deduced that central sensitization may behave as an amplifier of incoming peripheral neuronal information (6). More recently, studies on behaviour in relation to gene expression in the partial nerve injury model have revealed that two separate processes may be implicated in neuropathic pain. Thus, cold sensitive allodynia may develop in neurons, while an additional process including both neurons and immune cells instigates tactile allodynia (7).

The mechanisms underlying neuropathic pain and the experience of pain in general are far from complete in terms of understanding, though studies in humans and animal models have made a major contribution to this field. However, progress in this area of research has been hindered by innate translational problems firstly, in examining neuronal processes in humans; secondly, from changes in nociceptors brought about by invasive study methods; thirdly, because of the current inadequacy of existing techniques to record from microscopic structures involved in transduction of noxious stimuli; fourthly, due to the uncertainty that available animal models authentically reflect the perception of pain (8).

A selection of induced neuropathic pain models (9) and some subsequent common pain outcome measures (10) are shown in Table 1. In this connection, it has been noted that pain treatments targeting explicit pain mechanisms, tend to be more effective than disease/cause-based treatments which may be less specific. This may explain why many effective drugs in preclinical research fail in clinical trials (11). Also, the time course of pain development in animal neuropathic models and the duration of outcome assessments is inclined to be much shorter than that observed either in clinical trials or in clinical practice (11). These are important matters in extrapolating from bench to clinic. However, it should be kept in mind that among small endothermic animals such as rodents, the surface-to-volume ratio and basal metabolic rate are both greater than humans. In addition, in these smaller mammals, not only is the overall life span shorter, but cellular membranes are more polyunsaturated than larger species. Hence, there is a body size related variation 
Table 1 A selection of induced neuropathic pain models (9) and some more common examples of nociceptive outcome measures (10)

Models and measures Examples

Induced neuropathic pain models

Peripheral nerve Axotomy
injury models

Chronic sciatic nerve constriction

Partial sciatic nerve ligation

Spinal root ligation

Spared nerve injury

Excitotoxic models Chemotherapy-induced neuropathy

Alcohol-induced neuropathy

Diabetic: streptozotocin-induced neuropathy

Other neuropathic models

Post herpetic neuralgia

Pyridoxine-induced neuropathy

Trigeminal neuralgia (trigeminal nerve ganglion agar injection)

Orofacial pain (formalin injection in temporomandibular joint)

Nociceptive outcome measures

Reflexive outcome measures

Thermal

Hot-plate test

Hargreaves (control and test in the same subject)

Acetone-induced cold stimulus

Mechanical

von Frey filament application (static allodynia)

Brush (dynamic allodynia)

Pressure (Randall-Selitto test)

Non-reflexive outcome measures

Avoidance of
evoked stimul

Thermal escape test

Conditioned place preference/avoidance

Place escape avoidance paradigm

Behavioral function Eating, drinking, posture, gait, grooming, social interaction, general activity

in the membrane docosahexaenoic acid (DHA) content (12). Consequently, when considering the timeframe differences between studies with neuropathic models and clinical research, it is worthwhile bearing in mind these potentially influential allometric and biochemical factors.
Tissue and nerve damage lead to inflammation and initiation of repair processes triggering the condition of peripheral sensitization which is characterized by hyperexcitability and this usually subsides in the short-term upon healing. However, if the process of cellular insult is continuous as the result of a disease like diabetes, this state may be long lasting (11). In consequence, a neuropathic pain model based on this principle may prove to be useful. In view of this, the diabetic neuropathy model was developed (13).

In the same theme, longer-term neuropathic pain models such as chronic sciatic nerve constriction injury (14) and chemotherapy-induced peripheral neuropathy $(15,16)$ have proved useful in assessing potential activity versus neuropathiclike pain. Similarly, a number of diabetic neuropathic pain studies have been carried out using the diabetogenic $\beta$-pancreatic islet cell toxin streptozotocin (13). This technique has even been shown to be valuable in detecting activity of a drug-containing topical gel preparation not only against neuropathic-like allodynia but also vulvodynia $(17,18)$ and it has led to the prediction of clinical effectiveness (19). However, streptozotocin produces intense type 1 diabetic insulinopenia and hyperglycemia and because the type 2 form is more prevalent, this facet warrants further detailed examination. Moreover, the neuropathy incited by the two diabetic forms is fundamentally different since for example, strict glucose control has much higher efficacy in preventing type 1 rather than type 2 diabetic neuropathy (20). Consequently, a reliable neuropathic model for type 2 diabetes is exceptionally timely.

Recently, streptozotocin-induced experimental diabetes has been produced in neonatal rats (nSTZ) and it was demonstrated that in addition to glucose intolerance, the hyperglycemia induced in these animals was much milder than in adults (21). Also, C-fibre nerve conduction velocity in $\mathrm{nSTZ}$ animals was reduced in a similar fashion to adults, but it occurred considerably later in neonates following treatment with streptozotocin. In $\mathrm{nSTZ}$ animals, there was also a delayed augmentation of activated transcription factor 3 (ATF3) immunoreactivity in the spinal cord and the dorsal root ganglia (DRG) which correlated with an upregulation of both ATF3 and glial fibrillary acidic protein (GFAP). ATF3 is regarded as a marker of regeneration after injury in DRG neurons and GFAP is expressed by cells such as glial astrocytes in the central nervous system. It was reasonably suggested therefore that satellite glial cells, microglia and astrocytes are activated in the DRG and spinal cord following neonatal treatment with streptozotocin. It is 
noteworthy that all these characteristics were attended by tactile allodynia to von Frey filament application, substantiating the notion that $\mathrm{nSTZ}$ causes nerve injury. The nSTZ diabetic model has been designated as one which mimics type 2 diabetes (22), although more recent authors acknowledged that their findings did not fully support this overall postulation (21). Nevertheless, nSTZ did disclose some of the features of type 2 diabetes and the animals in the $\mathrm{nSTZ}$ model were much healthier than those in the type 1 diabetic model (STZ in adult animals). Consequently, the nSTZ model has been posited as a suitable choice for investigating diabetic neuropathic pain (21).

In the clinic, neuropathic pain of a spontaneous kind is undoubtedly more commonplace and distressing than the induced pain that is frequently measured as a neuropathic model outcome. In this respect, it has not been established whether induced pain in preclinical studies is accompanied by the perception of spontaneous pain in animals. Another confounding factor from a translational point of view, is that evoked pain outcome measures can account for the sensorydiscriminative element of pain often represented by ratings of intensity. However, they do not take into consideration the affective component of pain. This has been described as a characteristic reaction to a noxious stimulus heralded by an initial affective-motivational response sometimes termed "pain unpleasantness". A second affective stage, concomitant with longer-term pain involves intricate emotional consequences such as depression (23). It is evident therefore that pain outcome measures for useful neuropathic models such as $\mathrm{nSTZ}$ should mimic spontaneous pain which may have closer relevance to clinical practice (11). Current inclinations for pain assessment in neuropathic models have focused on more non-reflexive outcome measures in attempts to evaluate pain rather than nociceptive reflexes (24). Accordingly, the provision of animal locational choice may be employed, as in conditioned place aversion to noxious stimuli, or conditioned place preference to signify pain relief, although this latter paradigm may also manifest reward. Animal facial coding scales have been developed using an orbital tightening score to reflect the facial expression of pain level and vocalization has also been used to indicate an acute emotional aspect of pain. Other parameters such as cognitive function, sleep pattern, autonomic function and social interaction have also been studied from the perspective of chronic pain (24).

In the case of the $\mathrm{nSTZ}$ paradigm mentioned earlier, the generated tactile allodynia (associated with mild hyperglycemia, glucose intolerance, and glial cell activation) was relieved by gabapentin, metformin and morphine indicating the presence of neuropathic-like pain (21). Ostensibly, this model may well have translational value, though in consideration of the foregoing discussion, the model outcome measure that was employed, involved evoked pain rather than an affective component evaluation. This emphasizes the duality of translational challenges coupled to neuropathic models and their outcome measures, so clinically, not only does neuropathic pain remain refractory to treatment but also difficult to replicate and evaluate.

\section{Acknowledgements}

The author acknowledges valuable research collaboration with Professor Fazal Subhan, University of Peshawar, Pakistan.

\section{Footnote}

Conflicts of Interest: The author has no conflicts of interest to declare.

\section{References}

1. Woolf CJ, Mannion RJ. Neuropathic pain: aetiology, symptoms, mechanisms, and management. Lancet 1999;353:1959-64.

2. Dubin AE, Patapoutian A. Nociceptors: the sensors of the pain pathway. J Clin Invest 2010;120:3760-72.

3. Torrance N, Smith BH, Bennett MI, et al. The epidemiology of chronic pain of predominantly neuropathic origin. Results from a general population survey. J Pain 2006;7:281-9.

4. Bouhassira D, Lantéri-Minet M, Attal N, et al. Prevalence of chronic pain with neuropathic characteristics in the general population. Pain 2008;136:380-7.

5. Jensen TS, Finnerup NB. Allodynia and hyperalgesia in neuropathic pain: clinical manifestations and mechanisms. Lancet Neurol 2014;13:924-35.

6. Meacham K, Shepherd A, Mohapatra DP, et al. Neuropathic Pain: Central vs. Peripheral Mechanisms. Curr Pain Headache Rep 2017;21:28.

7. Cobos EJ, Nickerson CA, Gao F, et al. Mechanistic Differences in Neuropathic Pain Modalities Revealed by Correlating Behavior with Global Expression Profiling. Cell Rep 2018;22:1301-12.

8. Le Bars D, Gozariu M, Cadden SW. Animal models of 
nociception. Pharmacol Rev 2001;53:597-652.

9. Sousa AM, Lages GV, Pereira CL, et al. Experimental models for the study of neuropathic pain. Rev Dor 2016;17:S27-30.

10. Gregory NS, Harris AL, Robinson CR, et al. An overview of animal models of pain: disease models and outcome measures. J Pain 2013;14:1255-69.

11. Cohen SP, Mao J. Neuropathic pain: mechanisms and their clinical implications. BMJ 2014;348:f7656.

12. Hulbert AJ, Pamplona R, Buffenstein R, et al. Life and death: metabolic rate, membrane composition, and life span of animals. Physiol Rev 2007;87:1175-213.

13. Biessels GJ, Bril V, Calcutt NA, et al. Phenotyping animal models of diabetic neuropathy: a consensus statement of the diabetic neuropathy study group of the EASD (Neurodiab). J Peripher Nerv Syst 2014;19:77-87.

14. Shahid M, Subhan F, Ahmad N, et al. Topical gabapentin gel alleviates allodynia and hyperalgesia in the chronic sciatic nerve constriction injury neuropathic pain model. Eur J Pain 2017;21:668-80.

15. Shahid M, Subhan F, Ahmad N, et al. The flavonoid 6-methoxyflavone allays cisplatin-induced neuropathic allodynia and hypoalgesia. Biomed Pharmacother 2017;95:1725-33.

16. Ahmad N, Subhan F, Islam NU, Shahid M, Rahman FU, Sewell RDE. Gabapentin and its salicylaldehyde derivative alleviate allodynia and hypoalgesia in a cisplatin-induced neuropathic pain model. Eur J Pharmacol 2017;814:302-12.

Cite this article as: Sewell RD. Neuropathic pain models and outcome measures: a dual translational challenge. Ann Transl Med 2018;6(Suppl 1):S42. doi: 10.21037/atm.2018.09.58
17. Ali G, Subhan F, Abbas M, et al. A streptozotocin-induced diabetic neuropathic pain model for static or dynamic mechanical allodynia and vulvodynia: validation using topical and systemic gabapentin. Naunyn Schmiedebergs Arch Pharmacol 2015;388:1129-40.

18. Aman U, Subhan F, Shahid M, et al. Passiflora incarnata attenuation of neuropathic allodynia and vulvodynia apropos GABA-ergic and opioidergic antinociceptive and behavioural mechanisms. BMC Complement Altern Med 2016;16:77.

19. Hiom S, Patel GK, Newcombe RG, et al. Severe postherpetic neuralgia and other neuropathic pain syndromes alleviated by topical gabapentin. Br J Dermatol 2015;173:300-2.

20. Callaghan BC, Hur J, Feldman EL. Diabetic neuropathy: one disease or two? Curr Opin Neurol 2012;25:536-41.

21. Barragán-Iglesias P, Oidor-Chan VH, Loeza-Alcocer E, et al. Evaluation of the neonatal streptozotocin model of diabetes in rats: Evidence for a model of neuropathic pain. Pharmacol Rep 2018;70:294-303.

22. Takada J, Machado MA, Peres SB, et al. Neonatal streptozotocin-induced diabetes mellitus: a model of insulin resistance associated with loss of adipose mass. Metabolism 2007;56:977-84.

23. Horn C, Blischke Y, Kunz M, et al. Does pain necessarily have an affective component? Negative evidence from blink reflex experiments. Pain Res Manag 2012;17:15-24.

24. Barrot $M$. Tests and models of nociception and pain in rodents. Neuroscience 2012;211:39-50. 